\title{
Frontières
}

\section{La fin de vie en maison de repos (MR) et en maison de repos et de soins (MRS)}

\section{Gérard Marin}

Volume 24, numéro 1-2, automne 2011, printemps 2012

L’aide médicale à mourir

URI : https://id.erudit.org/iderudit/1013091ar

DOI : https://doi.org/10.7202/1013091ar

Aller au sommaire du numéro

Éditeur(s)

Université du Québec à Montréal

ISSN

1916-0976 (numérique)

Découvrir la revue

Citer cet article

Marin, G. (2011). La fin de vie en maison de repos (MR) et en maison de repos et de soins (MRS). Frontières, 24(1-2), 97-100. https://doi.org/10.7202/1013091ar d'utilisation que vous pouvez consulter en ligne.

https://apropos.erudit.org/fr/usagers/politique-dutilisation/ 


\section{La fin de vie en maison de repos (MR) et en maison de repos et de soins (MRS)}

Gérard Marin ${ }^{1}$

médecin de famille, président de l'Association francophone des médecins coordinateurs et conseillers en MRS et membre d'EOL (End of Life).

\section{MES CONVICTIONS}

$\mathrm{Au}$ fil de ma carrière longue déjà de 40 ans, je me suis forgé, à propos de la fin de vie, deux convictions fortes qui soutiennent ma pratique: la fin de vie constitue un tout, elle n'est pas séparée du reste de la vie; la fin de vie requiert une approche globale. Le médecin généraliste est un omnipraticien, il prend donc la fin de vie en charge de manière globale.

Je cite souvent cette phrase des Essais de Montaigne: "si nous avons besoin de sages-femmes pour nous mettre au monde, nous avons encore besoin d'hommes ou de femmes plus sages encore à nous en sortir». Il n'est pas précisé au médecin comment sortir de la vie, nous avons à aider les patients tout au long et en fin de vie. Or on constate souvent que des médecins ne tiennent pas jusqu'au bout la promesse qu'ils ont faite au malade de faire tout pour les aider, et se défilent quand les difficultés apparaissent en laissant leur patient au milieu du gué.

\section{MA PRATIQUE DES SOINS \\ PALLIATIFS}

Je pratique depuis longtemps les soins palliatifs. Pendant quarante ans, j'ai essayé de soulager le mieux possible mes patients en phase terminale, c'était là mon objectif principal. Pour ce faire, j'ai recouru sans culpabilité aux calmants, aux antidouleurs, voire aux neuroleptiques. Certes ces substances ont des effets secondaires, mais ceux-ci me paraissaient effectivement secondaires au regard de l'objectif principal. Pendant cette période, j'ai accompagné des phases terminales jusqu'au bout et en essayant de les soulager le mieux possible, y compris par l'administration de morphine que l'on utilisait cependant avec réticence. La peur que celle-ci suscite est-elle fondée ou non? Il est difficile de répondre de manière tranchée. Avec des dosages extrêmes, la mort pouvait survenir. Mais parfois aussi dans d'autres circonstances. Ainsi, au début de ma carrière, après cinq ans de pratique, j'ai vécu le cas d'une voisine atteinte d'un cancer du colon et qui souffrait énormément, mais sans qu'on puisse présager une mort imminente. Je lui ai administré une dose de spasmalgine (ancienne forme de morphine) qui l'a soulagée. Mais trente minutes plus tard, elle était morte, sans doute pas à cause de la morphine, mais en tout cas en toute quiétude. Pendant cette période, les médecins essayaient de faire au mieux pour soulager les souffrances physiques et aussi psychiques - car la mort fait toujours peur - avec les moyens du bord qui étaient fort réduits. À cette époque, nous ne pratiquions pas l'euthanasie, nous n'y étions pas formés.

Soeur Léontine ${ }^{2}$ et quelques autres ont ouvert des portes avec beaucoup de dynamisme. Nous leur devons beaucoup. Soeur Léontine connaissait beaucoup de monde. Elle a permis que les soins palliatifs auxquels elle s'était formée au RoyaumeUni arrivent en Belgique. Les soins pallia- 
tifs ont beaucoup apporté. D'abord, le fait de mettre absolument le patient au centre. Ensuite, des moyens, des possibilités matérielles, médicaments et techniques, telles que la possibilité de faire des injections sous-cutanées, ce que nous méconnaissions jusqu'alors. Les médecins intéressés par les soins palliatifs ont codifié de manière très précise l'administration de substances, leur moment, ils ont permis la familiarisation avec des cocktails inédits et que personne n'aurait osé administrer sans cette codification préalable.

Cependant, malgré des soins palliatifs correctement appliqués, certaines personnes continuaient à éprouver de très (ou de trop ?) grandes souffrances. Souffrir, ce n'est pas seulement avoir mal, c'est aussi se voir se dégrader, c'est se voir perdre sa dignité, c'est se sentir devenir invalide au sens premier du terme. La question s'est alors posée: peut-on entendre des demandes d'euthanasie? La loi ne le permettait pas. Il y eut sans doute des euthanasies clandestines parce que des médecins ont accepté de prendre le risque de soulager des patients définitivement, de les accompagner jusqu'au bout dans leur demande.

\section{MA PRATIQUE DE L'EUTHANASIE}

\section{L'APPORT DE LA LOI}

La loi est le résultat d'un long processus de maturation. Pour ceux qui voulaient accompagner leur patient jusqu'au bout selon la conviction de ce dernier (et non celle du médecin), elle fut un soulagement car elle créait un cadre juridiquement sécurisant tant pour le patient que pour le médecin. Le patient peut désormais demander l'euthanasie sans culpabilité. Le médecin peut accéder à cette demande dans les conditions posées par la loi. L'euthanasie doit être distinguée du suicide car elle n'est pas un acte désespéré, mais un acte réfléchi et posé sereinement. Elle était étrangère à ma culture générale, à ma culture médicale et à ma culture philosophique puisque j'avais été éduqué dans la religion catholique à laquelle j'adhérais avec conviction, même si aujourd'hui je ne pratique plus guère. Je n'ai eu aucune difficulté à entrer dans l'esprit de la loi : aider les gens à finir leur vie selon leur souhait, ce n'est pas tuer, c'est «donner» la mort.

\section{L'OPPOSITION STÉRILE ENTRE}

\section{SOINS PALLIATIFS ET EUTHANASIE}

On a souvent opposé soins palliatifs et euthanasie, ce qui est une très mauvaise idée, une erreur. Ils sont, l'un et l'autre, guidés par le respect dû aux gens qui font confiance à la personne à qui ils adressent leur demande. En effet, une

ON A SOUVENT OPPOSÉ SOINS PALLIATIFS ET EUTHANASIE, CE QUI EST UNE TRÈS MAUVAISE IDÉE, UNE ERREUR. ILS SONT, L'UN ET L'AUTRE, GUIDÉS PAR LE RESPECT DÛ AUX GENS QUI FONT CONFIANCE À LA PERSONNE À QUI ILS ADRESSENT LEUR DEMANDE.

demande d'euthanasie est une marque de confiance absolue, extrêmement émouvante pour le médecin: le patient confie sa vie au médecin et lui témoigne ainsi une confiance absolue. Aucune personne sensée ne se confie à quelqu'un qui va le tuer. L'euthanasie relève donc d'un tout autre registre que celui de tuer. Aider à mourir n'est pas tuer. C'est accompagner un patient en fin de vie et l'aider à finir celle-ci comme il le veut et quand il le veut. Ce qui est alors en jeu, c'est le respect de l'autonomie des patients, de la décision qu'ils prennent. Certains estiment que cette autonomie est réduite car les patients se trouvent dans une impasse, une voie sans issue. Mais j'estime que, même si l'horizon est bouché, demander l'euthanasie constitue un choix. Choisir, c'est renoncer. Et le renoncement ici est important. Certains patients choisissent de vivre leur souffrance jusqu'au bout. D'autres préfèrent demander que leur médecin y mette un terme. Ces patients restent toutefois peu nombreux: les décès par euthanasie s'élèvent à un très faible pourcentage de l'ensemble des décès, c'est donc un phénomène marginal, mais ô combien important pour celui qui le décide.

\section{LES EXIGENCES DÉONTOLOGIQUES ET LÉGALES}

Le code de déontologie - chapitre 9, articles 95 à 98 - a été modifié le 18 mars 2006, soit 4 ans après l'adoption de la loi. Un cheminement a donc été nécessaire. Les articles modifiés exigent que le médecin informe le patient en temps opportun de sa vie finissante, qu'il lui explique les initiatives qui peuvent être prises concernant la fin de vie, dont les soins palliatifs auxquels il a droit. Le médecin doit aussi informer son patient du soutien médical qu'il accepte de lui donner. Toutes ces informations doivent être données de manière claire et compréhensible pour le patient, ce qui n'est pas facile: il faut utiliser des termes adéquats, et souvent répéter les messages. Si le médecin ne dispose pas de connaissances suffisantes, il doit recueillir les avis nécessaires et/ou appeler un confrère compétent.

Le consentement libre et éclairé du patient aux décisions médicales en fin de vie est obligatoire, autant pour la mise en route de traitements que pour le retrait de traitements. Mais à propos de l'euthanasie, ce n'est pas de consentement qu'il s'agit: c'est le patient qui demande l'euthanasie; le médecin, lui, doit informer le patient. Quelques - rares - patients préfèrent ne pas savoir, ce que la loi sur les droits du patient leur permet. Dans ces cas-là, le médecin ne peut évidemment pas pratiquer l'euthanasie.

La loi sur l'euthanasie est bien faite. L'article 2 définit très précisément l'euthanasie: c'est l'acte pratiqué par un tiers qui met fin intentionnellement à la vie d'une personne à la demande de celle-ci. Il faut bien souligner ce qu'implique cette définition: sans demande explicite du patient, aucune euthanasie ne peut être pratiquée par le médecin.

\section{LE DEVOIR D'INFORMATION \\ DU MÉDECIN}

Le médecin doit-il informer son patient de la possibilité de l'euthanasie? Il doit l'informer en colloque singulier des différentes possibilités de gérer sa fin de vie. Doit-il être directif ou non? La réponse est difficile. Il doit informer de ce qui est possible, notamment de la possibilité des déclarations anticipées. En maison de repos et en maison de repos et de soins, on constate qu'il est difficile pour les assistantes et les assistants sociaux de demander aux patients s'ils ont pris des dispositions particulières par rapport à leur fin de vie. Les questions d'héritage, d'accompagnement spirituel, et de d'enterrement ou d'incinération ne soulèvent pas de problèmes, mais interroger les résidents sur leurs dispositions face à une possibilité de déclaration anticipée ou d'euthanasie est encore extrêmement difficile. J'estime que l'euthanasie doit être présentée dans l'ensemble des possibilités, soins palliatifs y compris. Mais ces derniers restent affectés d'une connotation négative: ils demeurent perçus comme fin des possibilités de traitement, comme fin de vie. Or dans ma pratique, j'ai rencontré des patients qui ont été parfois plusieurs fois en phase palliative. On pensait qu'ils étaient en fin de vie. Mais ils s'en sont sortis. Pour éviter cette connotation négative, on parle aujourd'hui de soins continus, de soins de confort. Mais ces diverses dénominations font qu'on ne sait plus bien de quoi on parle. Sans doute 
sont-elles liées à des manières différentes d'administrer les soins palliatifs. La toute grosse question est: comment annoncer un diagnostic difficile? On ne rencontre pas de problème, ni de peur avec l'annonce d'un infarctus qui peut tuer dans les minutes, voire les secondes qui suivent. Mais l'annonce d'un cancer qui ne tue pas pourtant immédiatement suscite une grande peur.

\section{EN MAISON DE REPOS (MR) ET MAISON DE REPOS ET DE SOINS (MRS)}

Comment la question se pose-t-elle en maison de repos et en maison de repos et de soins? On constate une évolution chez les assistantes et les assistants sociaux qui commencent à intégrer la possibilité d'interroger les résidents sur leur fin de vie. En droit, le directeur d'une maison de repos ou d'une maison de repos et de soins ne peut refuser que l'euthanasie soit pratiquée dans son institution. Cependant, pour que la pratique soit admise, il faut une préparation, un travail sur l'ambiance de la maison. Celui-ci se réalise par le biais de discussions, de symposiums. Ainsi, depuis 2002, le débat est en cours dans la MRS de Soignies et les institutions avoisinantes avec la participation du président de l'Ordre des médecins, de la responsable de la plate-forme soins palliatifs, de divers médecins qui pratiquent les soins palliatifs ou l'euthanasie. Des ateliers «tranche de vie» sur l'approche de la fin de vie sont en passe d'être réalisés avec les résidents. Ils seront axés sur la question: comment souhaitez-vous que se passe votre fin de vie? En effet, les personnes âgées ne parleront de leurs souhaits pour

s'exprime de manière ouverte ou déguisée, il faut dire aux soignants d'en parler au médecin qui doit lui-même en parler avec le patient. Le respect de la décision implique que le patient puisse clarifier sa demande. Celle-ci peut d'ailleurs changer lors de ces entretiens.

Les personnes âgées sont réceptives à ce genre de questions. Mais elles sont contraintes par le silence, par le tabou qui pèse sur la question de l'euthanasie. Après 80 ans, on constate qu'il y a très peu de demandes car les gros cancers détruisent beaucoup avant. En MR et MRS, les problèmes sont souvent cardiaques ou respiratoires mais on $\mathrm{y}$ rencontre moins de cancers. Toutefois, en 2009-2010, le rapport de la Commission fédérale de contrôle et d'évaluation de la loi relative à l'euthanasie indique que le nombre d'euthanasies pratiquées en maison de repos et en maisons de repos et de soins a doublé: il est passé de $4 \%$ à $8 \%$. Il faut noter que $50 \%$ environ des euthanasies se pratiquent au domicile, dont la MR ou la MRS qui sont le domicile institutionnel. Les euthanasies en MR et en MRS sont donc marginales. Leur nombre va-t-il augmenter? On ne peut le dire. Même si on peut supposer que oui car il est vraisemblable qu'il y aura davantage d'euthanasies pratiquées au nom d'une demande anticipée. Il faut se rappeler toutefois que la déclaration anticipée ne vaut qu'en cas d'inconscience. Certains patients sont dans cet état: des malades atteints d'Alzheimer ou des accidents vasculaires cérébraux qui ne peuvent plus s'exprimer. C'est pour eux que la demande anticipée est prévue.

\section{EN FAIT, LES QUESTIONS SE POSENT EN GRADATION :}

JE NE VEUX PLUS SOUFFRIR, JE SOUHAITE MOURIR,

\section{LAISSEZ-MOI MOURIR, FAITES-MOI MOURIR.}

la fin de leur vie que si on leur en parle, et ce que l'on constate, c'est que quand on leur en parle, elles veulent s'exprimer. Les ateliers leur permettent de s'exprimer sur différents sujets difficiles, par exemple aussi la sexualité qui n'est pas évidente en maison de repos. Le décès d'une jeune ergothérapeute a ralenti la mise en œuvre des ateliers «fin de vie» à Soignies. Dans cette maison de repos et de soins, il n'y a pas eu à ce jour de demande d'euthanasie réellement formulée. Il faut informer sur la procédure. En fait, les questions se posent en gradation: je ne veux plus souffrir, je souhaite mourir, laissez-moi mourir, faitesmoi mourir. Si cette dernière demande

\section{QUELLE LIMITE ? QUI LA PLACE ?}

La loi est-elle adéquate pour les patients atteints de maladies neuro-dégénératives, notamment l'Alzheimer? La déclaration anticipée a une validité réduite dans le temps: 5 ans. Or la maladie d'Alzheimer dure en moyenne 10 ans. L'exemple d'Hugo Claus (Van Neste, 2011/2012, note 6:) est celui de quelqu'un qui met sa limite à l'égard de la «souffrance inapaisable, insupportable et constante » qu'énonce la loi. Il faut demander aux gens jusqu'où ils acceptent d'aller. Ce sont eux qui mettent leur limite, pas le médecin. Les limites ne sont pas les mêmes pour un insuffisant cardiaque, un insuffisant respiratoire ou un malade atteint de dégénérescences neurologiques. Pour un patient myopathe ou atteint de dégénérescence musculaire, ce pourra être de ne plus boire son verre lui-même, pour un insuffisant respiratoire, ce pourra être de ne plus être capable d'aller de son lit à son fauteuil ou à table. C'est le patient qui fixe son seuil de tolérance. Mais si un patient n'a pas fait de déclaration anticipée, alors il faut l'accompagner jusqu'au bout. Selon les critères de la loi, on ne peut pratiquer l'euthanasie sans que le patient l'ait explicitement demandée. On risque autrement d'ouvrir la porte à des dérives inacceptables, voire des pratiques clandestines.

\section{LES SÉDATIONS TERMINALES}

Les sédations terminales constituent une prise en charge d'un processus menant à la mort sans que le patient l'ait demandé. Même si l'intention est bonne, cette pratique m'interpelle. Il faudrait en tout cas éteindre les querelles suscitées par ceux qui pratiquent des sédations terminales à l'encontre de ceux qui pratiquent une euthanasie. Ces derniers ne sont pas des criminels.

La question récurrente est celle du bienêtre du patient. C'est sa recherche qui doit soutenir les démarches du médecin.

\section{LES VALEURS QUI SONT AU CEUR \\ DE LA LOI ET LES QUESTIONS \\ QUI DEMEURENT}

Doit-on redouter que le temps avançant, les critères de la loi relative à l'euthanasie se distendent? Pour moi, j'en reviens toujours au caractère de la souffrance «inapaisable, intolérable et constante». À partir du moment où l'on intègre cela et que la demande du patient est répétée, on doit l'entendre et on peut répondre ou non à celle-ci, selon l'approche que l'on a. La loi met en avant la dignité de la personne, dont on se forge la signification soi-même. Y a-t-il des limites à cette autonomie? Je ne puis répondre que par des questions. Est-ce à nous de dire que le patient est capable de faire tel ou tel choix? Est-ce le soignant qui doit penser que le choix du patient est judicieux? Comment répondre dans le cadre des maisons de repos et des maisons de repos et de soins? Le médecin doit-il prolonger la vie à tout prix? Le patient a-t-il le droit de refuser un traitement approprié? La réponse est oui selon la loi sur les droits du patient. Le patient peut-il demander un traitement innovant? Il faut constater que de tels traitements donnent parfois de bons résultats. Qui décide de quoi ? On ne peut imposer à tous de décider par soi-même. Mais on ne peut pas empêcher ceux qui veulent décider par eux-mêmes de le faire. 
Notes

1. Ce texte a été rédigé par Marie-Luce Delfosse sur base d'un entretien avec Gérard Marin et est publié avec son accord.

2. Sœur Léontine, de la Congrégation des Sœurs hospitalières augustines, a été infirmière, puis médecin et licenciée en sciences hospitalières. Elle fut directrice générale de la Clinique Saint-Jean à Bruxelles de 1964 à 1988. Elle milita avec énergie et persévérance en faveur de la reconnaissance des soins palliatifs et mit sur pied en 1989 l'ASBL Soins palliatifs SaintJean qui permit la création du premier service de soins palliatifs en Belgique. Son livre $A u$ nom de la vie. Les soins palliatifs: éthique et témoignage (trad. du néerlandais par Guy Verwacht, Gembloux, Duculot, 1993) rend compte de ses convictions. Reconnue en Belgique comme une personnalité de grande envergure, sœur Léontine est décédée ce 19 février 2012. Voir aussi l'article de Nadia Huart «Soins palliatifs et euthanasie» dans ce numéro de Frontières. 